\title{
Nonavalent Human Papillomavirus Vaccine as a Treatment for Recalcitrant Warts in an Adult with HIV
}

\author{
Michael Tassavor, MD¹, Peter Hashim, MD¹, Folawiyo O. Babalola, BSA ${ }^{2}$, Mehul Bhatt, MD1 \\ ${ }^{1}$ Department of Dermatology, Icahn School of Medicine at Mount Sinai Hospital, New York, NY \\ ${ }^{2}$ University of Texas Health Science Center at San Antonio, San Antonio, TX
}

\section{ABSTRACT}

Cutaneous warts are seen in as many as a quarter of immunosuppressed patients. ${ }^{1}$ Cell-mediated immunodeficiency, as seen in HIV, is correlated with increased rates of palmoplantar warts. ${ }^{2}$ These warts often cause discomfort, and can progress to verrucous carcinoma. As they can be resistant to traditional treatments like cryotherapy, patients must resort to more aggressive and invasive measures like intralesional bleomycin. In this case report, we present an immunocompromised patient with HIV successfully treated for his palmoplantar human papilloma virus (HPV) warts using the nonavalent HPV vaccine (Gardasil $9^{\mathrm{TM}}$, Merck Inc, Kenilworth, NJ, USA) after only marginal improvement with six treatments of bleomycin.

\section{INTRODUCTION}

Cutaneous warts are verrucous lesions caused by the proliferation of HPV in the epidermis. The lesions are infectious and are thus highly prevalent in the general population. The majority of those affected are children, but the lesions can be observed in all ages. Immunocompromised patients have an increased risk of developing these lesions owing to their generalized increased susceptibility to infections. The consensus is that vaccinations, including the HPV vaccine, are primarily preventative measures. There have been limited studies on the use of HPV vaccinations as adjunct therapy to cutaneous warts in HIV patients. We report a case of an HIV patient successfully treated with HPV vaccination after multiple treatment failures.

\section{CASE PRESENTATION}

A 56-year-old white man with known HIV on treatment (undetectable viral load) presented to Dermatology with a six-year history of palmoplantar warts. He reported failure of multiple rounds of cryotherapy, electrodessication and imiquimod cream under the supervision of his previous dermatologist. On physical exam, the patient had more than 21 verrucous plaques of various sizes on the soles of his feet bilaterally as well as the palmar surface of thumb of his left hand (Figure 1). Aside from the above, his medical history was significant for spondyloarthritis on sulfasalazine, and $\mathrm{HCV}$, which resolved spontaneously in the distant past. He was tried on one intralesional candida injection and one round of cryotherapy with inadequate response. 


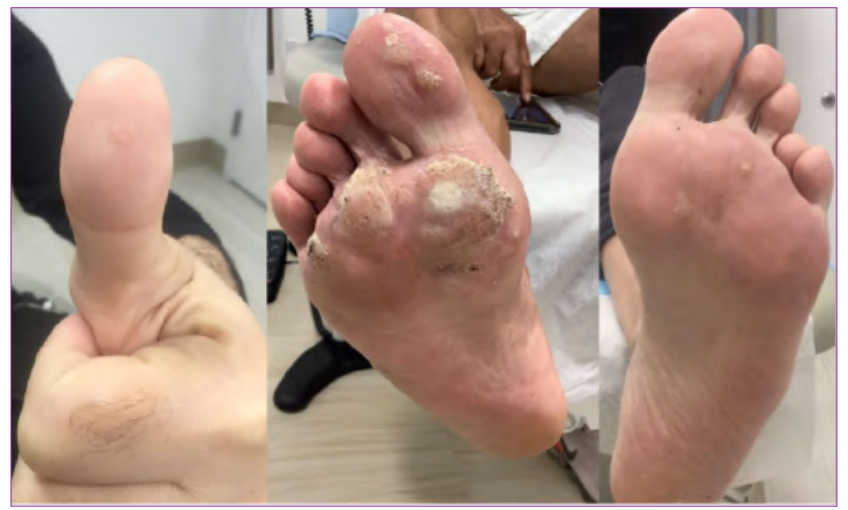

Figure 1. Before treatment with bleomycin

Six treatments of intralesional bleomycin were attempted with mild improvement in the injected areas (Figure 2). His PCP started him on the nonavalent HPV vaccine regimen before his seventh bleomycin injection, and by his eighth visit roughly two months later, he was noted to have a dramatic reduction in all lesions, including those that had not been injected with bleomycin. By his ninth visit three months after his first dose of the HPV series, his left sole had continued to clear rapidly, his right sole was clear except for a single small lesion, and his thumb had completely cleared (Figure 3). Despite the results, the patient desired to continue aggressive treatment and the few remaining lesions were injected with bleomycin once more.

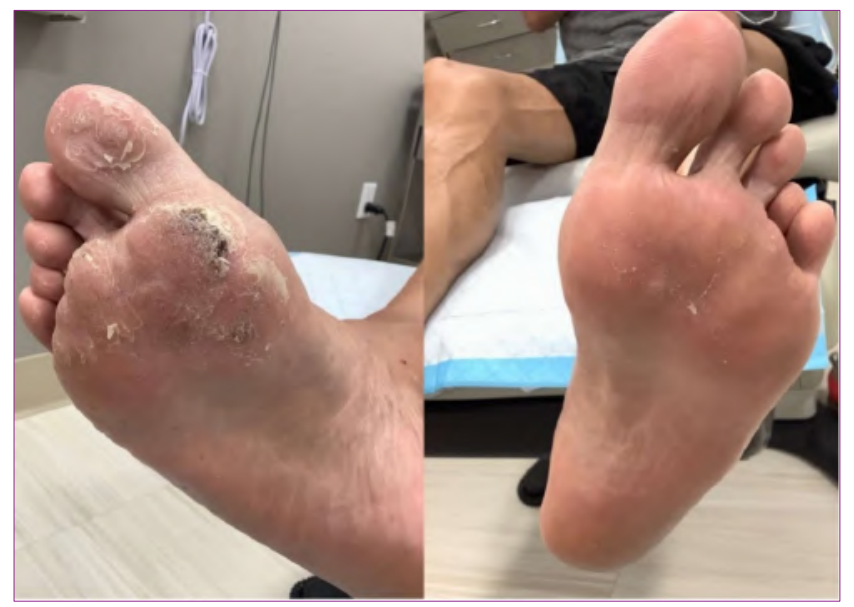

Figure 2. After 5 injections of bleomycin, before first HPV shot

\section{DISCUSSION}

Common cutaneous warts are classically caused by HPV strains 1,2 , and 4 , though it is known that immunosuppressed patients with plantar warts are more likely to have atypical HPV types like HPV-69. ${ }^{3,4}$ They are easily acquired via contact with the viral particle and spread through autoinoculation to different areas. Treatment of verruca include cryotherapy, salicylic acid, imiquimod, 5-flourouracil, cantharidin, intralesional Candida antigen, intralesional bleomycin, oral cimetidine, photodynamic therapy, and pulsed dye laser. None are universally effective.

Intralesional bleomycin is the treatment of choice at our institution for recalcitrant warts. This patient, with at least six years history of disease, had only marginal improvement with six previous injections before dramatically improving after a single shot of the vaccine, including areas we had not treated with bleomycin. This suggests the clinical improvement came from the vaccine rather than the bleomycin or spontaneous clearance. The vaccine series was started for prophylactic reasons and was only noted incidentally. This phenomenon has been reported before in immunosuppressed patients ${ }^{5-8}$, but not yet in patients with HIV. A recent single center retrospective cohort study $(n=16$, two immunocompromised patients, one lost to follow up, the second deceased due to unrelated reasons) of recalcitrant warts showed nearly $50 \%$ response to HPV vaccination, comparable to conventional treatments. The authors noted no relationship in response to number of prior treatments, age, or anatomic location. ${ }^{9}$ 


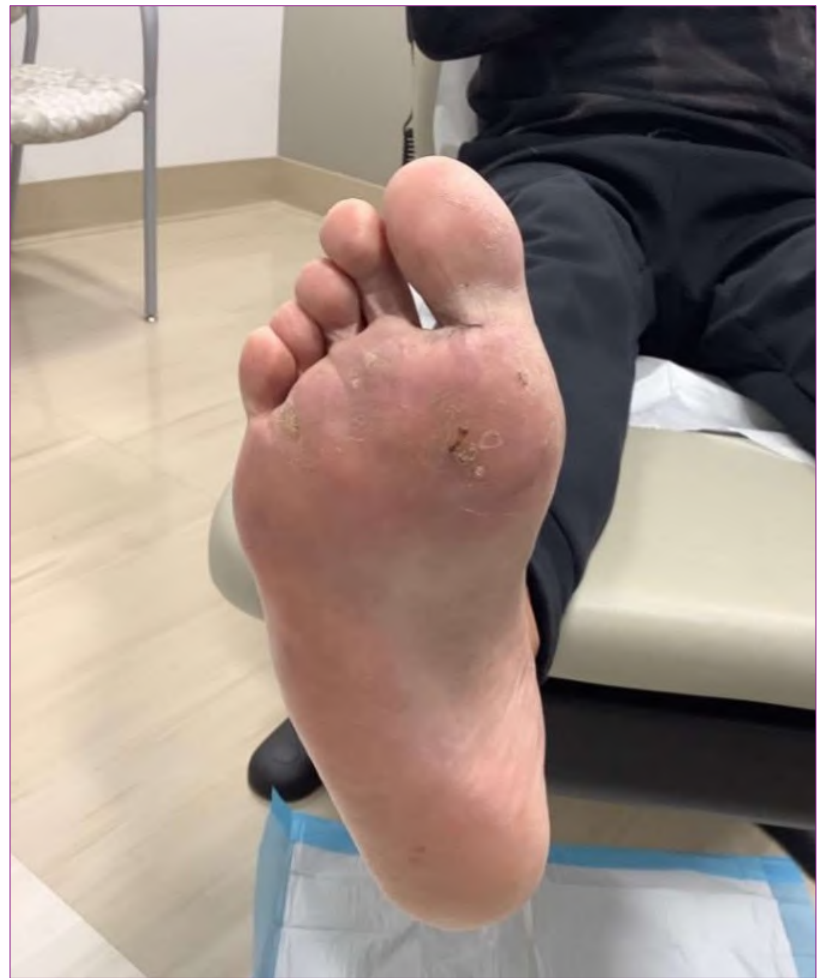

Figure 3. After 8 injections of bleomycin, 3 months after first HPV shot

\section{CONCLUSION}

While there are three FDA approved vaccines, only the nonavalent Gardasil 9 is currently available today in the United States, targeting HPV 6, 11, 16, 18, 31, 33, 45, 52 and 58. This vaccine does not cover the strains commonly associated with cutaneous warts, suggesting cross-reactivity with other HPV strains as has been shown before. ${ }^{10}$ With an excellent safety profile, proven efficacy against nearly $90 \%$ of HPV associated cancer, and insurance coverage up to 45 years of age in adults, the nonavalent HPV vaccine may represent an early and relatively non-invasive adjunct to first line therapy. Our patient improved after numerous failed trials with bleomycin, including in untreated areas, supporting vaccination as an effective adjunct therapy. As vaccination rates increase, it may also change annual incidence of these lesions.
Conflict of Interest Disclosures: None

Funding: None

Corresponding Author:

Michael Tassavor, MD

Icahn School of Medicine at Mount Sinai

325 W 15th St

New York, NY 10011

Phone: 212-367-0145

Email: mtassavor2@gmail.com

\section{References:}

1.Wieland $U$, Kreuter A, Pfister H. Human papillomavirus and immunosuppression. Curr Probl Dermatol. 2014;45:154-65. doi:10.1159/000357907

2.Leto M, Santos Júnior GF, Porro AM, Tomimori J. Human papillomavirus infection: etiopathogenesis, molecular biology and clinical manifestations. An Bras Dermatol. Mar-Apr 2011;86(2):306-17. doi:10.1590/s0365-05962011000200014

3.King CM, Johnston JS, Ofili K, et al. Human papillomavirus types 2,27 , and 57 Identified in plantar verrucae from HIV-positive and HIVnegative individuals. J Am Podiatr Med Assoc. Mar 2014;104(2):141-6. doi:10.7547/0003-0538104.2.141

4.Whitaker JM, Palefsky JM, Da Costa M, King CM, Johnston JS, Barbosa P. Human papilloma virus type 69 identified in a clinically aggressive plantar verruca from an HIV-positive patient. J Am Podiatr Med Assoc. Jan-Feb 2009;99(1):8-12. doi:10.7547/0980008

5.Ferguson SB, Gallo ES. Nonavalent human papillomavirus vaccination as a treatment for warts in an immunosuppressed adult. JAAD Case Rep. Jul 2017;3(4):367-369. doi:10.1016/j.jdcr.2017.05.007

6.Smith SP, Baxendale HE, Sterling JC. Clearance of recalcitrant warts in a patient with idiopathic immune deficiency following administration of the quadrivalent human papillomavirus vaccine. Clin Exp Dermatol. Apr 2017;42(3):306-308. doi:10.1111/ced.13038

7.Silling S, Wieland U, Werner M, Pfister $\mathrm{H}$, Potthoff A, Kreuter A. Resolution of novel human papillomavirus-induced warts after HPV vaccination. Emerg Infect Dis. Jan 2014;20(1):1425. doi:10.3201/eid2001.130999

8. Kreuter A, Waterboer T, Wieland U. Regression of cutaneous warts in a patient with WILD syndrome following recombinant quadrivalent human papillomavirus vaccination. Arch Dermatol. Oct 2010;146(10):1196-7.

doi:10.1001/archdermatol.2010.290

July 2021 Volume 5 Issue 4 
9.Waldman A, Whiting D, Rani M, Alam M. HPV Vaccine for Treatment of Recalcitrant Cutaneous Warts in Adults: A Retrospective Cohort Study. Dermatol Surg. Dec 2019;45(12):1739-1741. doi:10.1097/dss.0000000000001867

10.Landis MN, Lookingbill DP, Sluzevich JC.

Recalcitrant plantar warts treated with recombinant quadrivalent human papillomavirus vaccine. J Am Acad Dermatol. Aug 2012;67(2):e73-4. doi:10.1016/j.jaad.2011.08.022 